\title{
Tecnología lítica en la transición de cazadores recolectores a sociedades agropastoriles en la porción meridional de los Andes Centro Sur
}

SALOMÓN HoCSMAN ${ }^{1}$

\section{RESUMEN}

En este trabajo se abordan las transformaciones en la tecnología lítica producidas durante la transición de grupos cazadores recolectores a sociedades agropastoriles en la porción meridional de los Andes Centro Sur. Específicamente, se analizan las variaciones diacrónicas en las frecuencias de artefactos formatizados tallados recuperados en sitios arqueológicos de Antofagasta de la Sierra (Puna Meridional argentina), fechados entre los 5500 y los 1500 años AP, en función de la estimación de la inversión de tiempo y energía en la confección de los instrumentos. Por otro lado, se compara con información proveniente de la Puna Septentrional argentina y su borde, y del borde de puna del desierto del norte de Chile. Al respecto, se exploran aspectos vinculados con el proceso de toma de decisiones y su vinculación con el cambio tecnológico.

Palabras claves: tecnología lítica - transición cazadores recolectores a sociedades agropastoriles - porción meridional de los Andes Centro Sur.

\section{ABSTRACT}

Changes in lithic technology that occurred during the transition from hunter-gatherers to agro-pastoralist societies in southern Andes Centro Sur, are studied. Specifically, diachronic variations in flaked stone tools frequencies recorded in archaeological sites of Antofagasta de la Sierra (Southern Argentinean Puna) between 5500-1500 years $B P$, are analyzed. Time and energy investment in the manufacture of lithic tools, are utilized. Variations in tool frequencies are compared with archaeological data from Northern Argentinean Puna and its border, and from the puna border of the Northern Desert of Chile. Aspects of decision-making processes related to technological change, are explored.

Key words: lithic technology - transition from huntergatherers to agropastoralist societies - southern South Central Andes.

Recibido: julio 2005. Aceptado: marzo 2006

1 CONICET - Instituto de Arqueología y Museo, Facultad de Ciencias Naturales e Instituto Miguel Lillo, Universidad Nacional de Tucumán. San Martín 1545 (4000) San Miguel de Tucumán, ARGENTINA. Email: shypb@arnet.com.ar

\section{Introducción}

En coincidencia con el proceso que llevó de un modo de vida cazador recolector predominante a otro agropastoril, se produjo una serie de transformaciones tecnológicas que modificaron drásticamente la forma en que se estructuraban los conjuntos de artefactos líticos tallados.

En este trabajo se abordan dichos cambios, de acuerdo a dos objetivos: 1) analizar las variaciones diacrónicas en las frecuencias de artefactos formatizados tallados recuperados en contextos arqueológicos de la microrregión de Antofagasta de la Sierra (Puna Meridional argentina), ubicados por dataciones radiocarbónicas entre los 5500 y los 1500 años AP, a base de la estimación de la inversión de trabajo en la confección del instrumental lítico, y 2) comparar con información proveniente de otras áreas de la porción meridional de los Andes Centro Sur.

Puesto que el principal interés es analizar la variabilidad tecnológica y tipológica de los conjuntos de artefactos líticos en el proceso de cambio de los grupos cazadores recolectores a agropastoriles en la Puna Meridional argentina (Hocsman 2002 Ms), la aplicación de las propuestas y premisas de la arqueología evolucionista darwiniana, centradas en la variación (Dunnell 1989; Lyman y O’Brien 1998; Boone y Smith 1998), constituye una vía pertinente de aproximación. De esta forma, se propone trabajar desde uno de los enfoques que definen a dicha corriente teórica, la ecología evolutiva o ecología del comportamiento (Bettinger 1991; Winterhalder 2001; Winterhalder y Smith 1992). Específicamente, interesan de esta perspectiva aquellos aspectos vinculados con el proceso de toma de decisiones y su vinculación con el cambio tecnológico.

Si bien el énfasis está puesto en el análisis de las variaciones diacrónicas de los artefactos formatizados en Antofagasta de la Sierra, se considera también información complementaria procedente de contextos 
arqueológicos de la Puna Septentrional argentina y su borde oriental, y del borde de puna del desierto del norte de Chile (Figura 1), datados entre 5000 y 2000 años AP, aproximadamente.

\section{Las evidencias arqueológicas en Antofagasta de la Sierra (5500-1500 AP)}

La secuencia arqueológica local

De acuerdo a Yacobaccio (1998), desde los 5000 años AP, aproximadamente, se incrementan notablemente las evidencias de ocupación humana en la puna argentina. La microrregión de Antofagasta de la Sierra presenta una situación coincidente, ya que al analizar las cantidades de sitios datados sobre la base de cronologías absolutas y relativas, se observa que la proporción de ocupaciones entre el 9500 y el 5500 AP es considerablemente menor en relación al momento 5500-2000 AP. Pero no sólo es mayor la cantidad de sitios en este último momento, sino que también aumenta el número de los mismos con ocupaciones relativamente sincrónicas (Hocsman 2002). Esta tendencia creciente continúa a partir del 2000 AP, con las ocupaciones agropastoriles, de acuerdo a la información recuperada en el fondo de cuenca, sectores intermedios y quebradas de altura (Olivera 1992; López Campeny 2001 Ms).

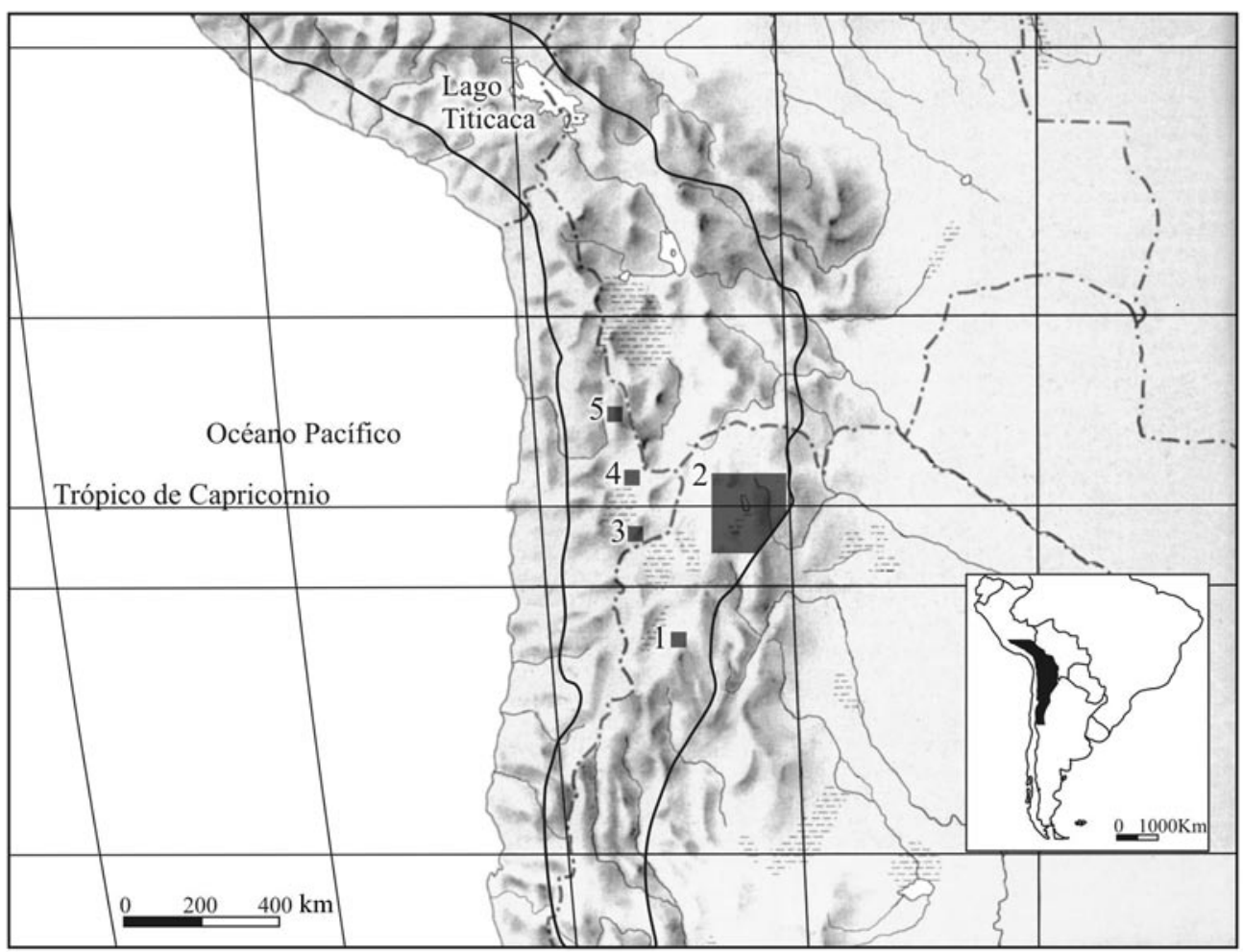

Referencias:

Area de Puna

Localidad arqueológica

1 Antofagasta de la Sierra

2 Sector considerado de Puna Norte y su borde oriental

3 Quebrada de Tulan

4 Quebrada de Puripica

5 Alto Loa

Figura 1. Ubicación de localidades arqueológicas. 
Ahora bien, los sitios y fechados radiocarbónicos disponibles señalan un vacío de información, hasta el momento, en lo que respecta a ocupaciones domésticas o "sitios de habitación", entre los 3200 y los 2100 años AP (Hocsman 2002) (Figura 2). Esto, se remarca, no significa un hiato de ocupación en Antofagasta de la Sierra, ya que se cuenta con dataciones de $2480 \pm 60$ AP en Quebrada Seca 3 y $2870 \pm 40$ AP en Cueva Cacao 1A, además de recurrentes dataciones entre los 3000 y $3500 \mathrm{AP}$ (Punta de la Peña 11 y Cueva Cacao 1A), aunque se trata de contextos funerarios y/o rituales que no cuentan con los materiales líticos necesarios para el tipo de análisis aquí desarrollado. A esto se debe sumar el hecho de que todos los sitios de habitación entre 5500 y 3000 AP se presentan en cuevas y aleros, surgiendo el interrogante de la ausencia de campamentos a cielo abierto.

Los sitios considerados y su cronología

Se consideraron para el análisis las siguientes bases residenciales, de acuerdo a su situación cronológica
(Cuadro 1) y a la presencia de materiales líticos factibles de ser analizados:

- Quebrada Seca 3 (QS3): Abrigo rocoso (cueva) situado en las quebradas de altura. ${ }^{2}$ Los artefactos formatizados analizados provienen de los niveles 2b1 a 2b5 (Aschero et al. 1991, 199394), datados entre los 5500 y los 4500 años AP. Se incluyeron aquí los artefactos formatizados recuperados en todas las campañas realizadas hasta el momento.

- Punta de la Peña 4 (PP4): Abrigo rocoso (alero) localizado en los sectores intermedios (Toselli 1998 Ms). Los instrumentos considerados proceden de la excavación realizada en el año 1996, incluyéndose todas las capas correspondientes a cazadores recolectores. Los fechados radiocarbónicos disponibles enmarcan a las ocupaciones del Holoceno Tardío de este sitio entre los 4100 y los 3200 años AP.

\begin{tabular}{|c|c|c|c|c|c|}
\hline Sitio & Capa/nivel & $\begin{array}{c}\text { Datación } \\
\text { radiocarb (AP) }\end{array}$ & Lab. $\mathbf{N}^{\circ}$ & Material & Fuente \\
\hline \multirow[t]{6}{*}{ QS3 } & $2 \mathrm{~b} 1$ & $5400 \pm 90$ & LP-270 & carbón & Pintar 1996 \\
\hline & $2 \mathrm{~b} 2$ & $4410 \pm 60$ & UGA-8357 & hueso & Aschero com pers. 2003 \\
\hline & $2 \mathrm{~b} 2$ & $4510 \pm 100$ & B-27801 & gramíneas & Aschero et al. 1991 \\
\hline & $2 \mathrm{~b} 2$ & $4930 \pm 110$ & AC-1115 & carbón & Aschero et al. 1991 \\
\hline & $2 \mathrm{~b} 3$ & $4770 \pm 80$ & B-27802 & carbón & Aschero et al. 1991 \\
\hline & $2 \mathrm{~b} 5$ & $5380 \pm 70$ & B-59927 & carbón & Aschero et al. 1993/94 \\
\hline \multirow[t]{5}{*}{ PP4 } & $3 x$ & $3820 \pm 100$ & UGA-9254 & carbón & Aschero com pers. 2003 \\
\hline & $4 \mathrm{a}$ & $3870 \pm 90$ & B-77748 & madera & Pintar 1996 \\
\hline & $4 \mathrm{~b}(1)$ & $4060 \pm 90$ & B-77749 & madera & Pintar 1996 \\
\hline & $5(6) 2$ & $3250 \pm 50$ & UGA-8354 & hueso & Aschero com pers. 2003 \\
\hline & $5(6) 2$ & $4100 \pm 160$ & UGA-7976 & carbón & Aschero com pers. 2003 \\
\hline \multirow[t]{2}{*}{ PCh1.1 } & $3^{\circ}$ ext. & $3590 \pm 55$ & LP-263 & carbón & Pintar 1996 \\
\hline & $4^{\circ}$ ext. & $3660 \pm 60$ & LP-261 & carbón & Pintar 1996 \\
\hline \multirow[t]{7}{*}{ CChM1 } & Nivel III & $1670 \pm 60$ & B-27199 & carbón & Olivera 1992 \\
\hline & Nivel IV & $1660 \pm 60$ & LP-251 & carbón & Olivera 1992 \\
\hline & Nivel Vc & $1530 \pm 70$ & B-27201 & carbón & Olivera 1992 \\
\hline & Nivel VII & $1740 \pm 60$ & B-27202 & carbón & Olivera 1992 \\
\hline & Nivel VII & $1930 \pm 70$ & B-27200 & carbón & Olivera 1992 \\
\hline & Nivel VIII & $2120 \pm 60$ & LP-299 & hueso & Olivera 1992 \\
\hline & Nivel IX & $1440 \pm 100$ & LP-295 & hueso & Olivera 1992 \\
\hline
\end{tabular}

Cuadro 1. Dataciones radiocarbónicas de los sitios considerados.

2 Se emplea la diferenciación ambiental de Olivera (1992) entre fondo de cuenca, sectores intermedios y quebradas de altura. 
- Peñas Chicas 1.1 (PCh1.1): Abrigo rocoso (alero) situado en los sectores intermedios (Pintar 1996). Se consideraron los materiales obtenidos durante las campañas de enero y mayo/junio de 1991. El sitio ha sido datado entre 3600 y 3500 AP.

- Casa Chávez Montículos 1 (CChM1): Sitio a cielo abierto ubicado en el fondo de cuenca, compuesto por estructuras monticulares adscribibles a ocupaciones agropastoriles (Olivera 1992). El análisis de los materiales fue realizado por Escola (2000). Se tuvieron en cuenta todas las capas, datadas entre 2100 y 1500 AP.

\section{Definición de grupos cronológicos}

Se propone el análisis de las muestras de artefactos formatizados de los contextos arqueológicos considerados a base de la definición de unidades temporales a partir de criterios estrictamente cronológicos (Borrero 1993). Al respecto, se sigue la propuesta de Yacobaccio y Guraieb (1994) de conformación de patrones de agrupamiento de fechados radiocarbónicos.

De esta forma, se establecieron grupos cronológicos (GC1) cuyo criterio de definición se basa en conjuntos de fechas que superponen sus rangos en un sigma (Cuadro 1, Figura 2). Como criterio complementario se utiliza la posición relativa de las fechas disponibles y de los niveles estratigráficos.

Se conformaron de esta manera tres grupos cronológicos (Cuadro 2). La diferencia cronológica es notoria entre el GC2 y el GC3, pero mínima entre el GC1 y el GC2. Esta última, no obstante, se considera relevante, puesto que hay una diacronía de 300 años entre la fecha más tardía del GC1 en relación a la más temprana del GC2, sin tener en cuenta los sigmas; uno de los cuales, por un lado, presenta una desviación estándar importante. Por otro, cabe destacar que en el GC1, a pesar de no haber superposición entre las fechas anteriores al $5300 \mathrm{AP}$ y posteriores al $4900 \mathrm{AP}$ (Figura 2), el hecho de contar con un nivel no directamente datado (2b4), pero que ha sido situado estratigráfica y cronológicamente entre las dataciones correspondientes a los niveles 2b3 y 2 b5 por Aschero y colaboradores (1993-94), estaría señalando una secuencia ininterrumpida. Al considerar el GC2, si bien el fechado de 3200 AP no presenta superposición con las dataciones restantes, por cercanía relativa se incluye al mismo dentro de este grupo. Idéntica decisión se toma para las dos fechas más tempranas del GC3.

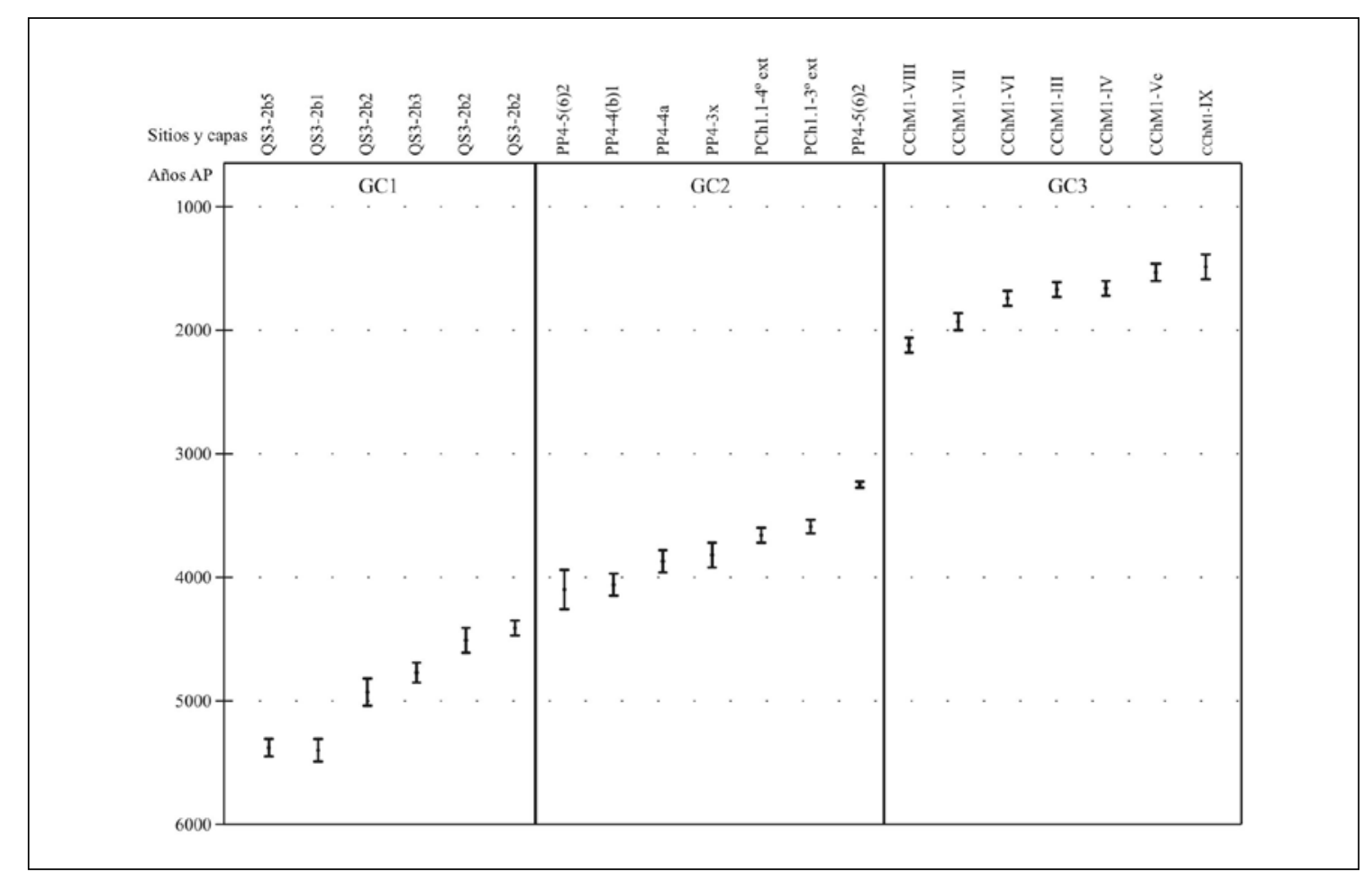

Figura 2. Distribución de dataciones radiocarbónicas para los sitios considerados. 


\begin{tabular}{|ccccc|}
\hline Grupos & Media (AP) & $\mathbf{N}^{\mathbf{3}}$ & Rango $^{\mathbf{4}}$ & Sitios \\
\hline 1 & 4900 & 6 & $5490-4350$ & QS3 \\
2 & 3764 & 7 & $4260-3200$ & PP4 - PCh1.1 \\
3 & 1727 & 7 & $2180-1340$ & CChM1 \\
\hline
\end{tabular}

Cuadro 2. Características de los grupos cronológicos. ${ }^{3}$ Cantidad de fechados radiocarbónicos. ${ }^{4}$ Un sigma.

Con el objeto de establecer la validez de la discriminación en tales grupos cronológicos, se aplicó el análisis de varianza (ANOVA) para controlar la existencia de diferencias significativas entre las medias de cada grupo. Para ello, se empleó el programa informático InfoStat, desarrollado por la Cátedra de Estadística y Biometría de la Facultad de Ciencias Agropecuarias de la Universidad Nacional de Córdoba.

A partir de dicha técnica es posible sostener que hay variaciones significativas entre las medias de los conjuntos de fechados de los grupos cronológicos definidos (ANOVA: $\mathrm{F}=166,62 ; \mathrm{p}<0,001$ ). Por otra parte, se aplicó el Test de Tukey por medio del programa informático mencionado para definir entre qué grupos había diferencias, resultando en la presencia de diferencias significativas entre los tres $(\mathrm{p}<0,001)$.

\section{Una aproximación a la tecnología lítica desde la ecología evolutiva}

Toma de decisiones, elección racional y tecnología lítica

La ecología evolutiva, cuyo interés es analizar la persistencia diferencial de la variabilidad en el comportamiento a lo largo del tiempo, entiende a los cambios en el comportamiento como formas de adaptación fenotípica a condiciones sociales y ecológicas variables, partiendo del supuesto de que la selección natural ha diseñado a los organismos para responder a las condiciones locales de manera de incrementar su fitness (Smith y Winterhalder 1992; Boone y Smith 1998).

De esta forma, los grupos humanos tenderían a seleccionar ciertos comportamientos, cuyo efecto neto es maximizar su fitness reproductivo o inclusivo dentro de un rango de alternativas. Dicha selección tendría lugar en un contexto que presupone no sólo un conjunto de objetivos generales, sino también preferencias por estrategias con alto grado de probabilidad de llevar a cabo tales objetivos. En este contexto, el proceso de selección natural es el responsable de decidir, mantener y alterar tales objetivos y preferencias (Smith y Winterhalder 1992; Kelly 1995).

Así, se asume que la selección favorece aquellos rasgos con fitness elevado y que la gente evalúa los fenotipos disponibles y adopta aquellos con alta utilidad. En suma, se espera que se seleccionen estrategias que cumplan mejor los objetivos propuestos que otras existentes. Es decir, que se opte por comportamientos que tiendan hacia la optimización (Bettinger 1991).

El proceso de toma de decisiones implica, entonces, una elección racional. De acuerdo a Smith y Winterhalder (1992), el término racional no refiere a los fines, sino a la relación entre fines y medios. Por lo tanto, actores racionales son aquellos que persiguen sus fines (cualesquiera que sean) tan efectivamente como sea posible y que no cometen errores lógicos al ordenar sus preferencias, lo cual significa que tienen en cuenta las posibles consecuencias de cada uno de los cursos de acción abiertos a ellos (Jochim 1976).

Siguiendo a Bousman (1993), la tecnología puede tener un rol crítico en determinar las elecciones económicas de los grupos humanos, por lo que deberían integrarse a los análisis los costos y beneficios que conlleva la utilización de variantes tecnológicas específicas.

Es así que la tecnología lítica (sensu Bleed 1997) puede vincularse a los análisis de optimización mediante la consideración de los costos de producción de distintas alternativas tecnológicas, a partir de la estimación de la inversión de trabajo, en tiempo y energía, en la confección de artefactos líticos tallados. De esta forma, las estrategias tecnológicas disponibles conforman un rango de opciones con costos y beneficios variables (Hayden et al. 1996; Andrefsky 1998; Cowan 1999; Tomka 2001) que definen tasas de retorno particulares, con sus consiguientes consecuencias sobre el fitness. 
Categorías analíticas empleadas

La documentación de la variación requiere de una aproximación que focalice más en el comportamiento de atributos a lo largo del tiempo, que en la construcción de tipos (Kelly 2000). Por tal motivo, se parte de una clasificación de artefactos formatizados basada, por un lado, en la presencia de adelgazamiento, reducción o trabajo no invasivo (ex marginal), $y$, por el otro, si se trata de formatización bifacial o unifacial, siguiendo criterios definidos por Aschero y Hocsman (2004 Ms) (Cuadro 3). Dichas categorías configuran clases técnicas a base de la observación, en los artefactos formatizados, de la cobertura total o parcial de los lascados y de la presencia o ausencia de rebaje en el espesor de las piezas, en cada una de sus caras; del tipo de sección en norma sagital y lateral y de su tamaño y espesor relativos.

Tanto en el adelgazamiento como en la reducción se afectan proporcionalmente más las caras que los bordes. En el primero hay una doble intención, por un lado, reducir el espesor de un artefacto y, por el otro, mantener con la menor afectación posible el ancho, mientras que en la segunda el rebaje tiene el objetivo de buscar conformaciones específicas de contornos y/o secciones. Ahora bien, ambas categorías se pueden diferenciar, entre otros aspectos, porque en el adelgazamiento: a) los lascados se extienden desde el borde hasta un poco más allá del centro del artefacto, y b) contactan o se superponen con negativos provenientes del margen opuesto, afectando el espesor; en la reducción, en cambio, si bien los negativos de lascado pueden llegar al centro de la cara, la tendencia es: a) que no alcancen a cubrir el centro de la cara o, en el caso de alcanzarlo, b) que no se superpongan con los lascados procedentes del otro borde (Aschero y Hocsman 2004) (Figura 3).

El trabajo no invasivo, por su parte, tiende a afectar sólo los bordes de la pieza, con escasa o nula modificación del centro de la cara o caras. Refleja una intención de búsqueda de una morfología de filo o punta particular, con ciertos requerimientos en cuanto de bisel, ángulo y forma de su sección, delineación del filo, etc. (Aschero y Hocsman 2004) (Figura 3).

En lo que respecta a las nociones de bifacial y unifacial, se considera la propuesta de Aschero (1983 Ms).

\section{Clases técnicas y costos de producción}

Un punto clave a la hora de considerar a las clases técnicas es que cada una de ellas implica un grado diferente de inversión de trabajo en la manufactura, que se ve reflejada en la morfología. Asimismo, presentan requerimientos variables de habilidad y destreza técnica. Sobre esta base se puede establecer un continuиm en la morfología de los artefactos que pueden ser producidos con muy poco esfuerzo frente a la de otros que requieren de un gran esfuerzo de producción.

Se enumeran a continuación las clases técnicas consideradas en este trabajo, en función de una inversión de trabajo decreciente: adelgazamiento bifacial, adelgazamiento unifacial, reducción bifacial, reducción unifacial, trabajo no invasivo bifacial y unifacial (modificado de Aschero y Hocsman 2004).

Es fácilmente discernible que el adelgazamiento y la reducción requieren de un mayor esfuerzo de manufactura y habilidad que el trabajo no invasivo, dada la insistencia de ambas estrategias sobre la superficie de la o las caras, a diferencia de esta última, que se limita al tratamiento de los bordes.

Ahora bien, al comparar el adelgazamiento con la reducción, el primero implica una mayor inversión de tiempo y energía de producción, debido a que requiere que las extracciones lleguen y excedan al centro de la cara. Para que esto sea posible, es

\begin{tabular}{|c|c|c|c|c|c|c|c|c|c|}
\hline Sitio & $\begin{array}{c}\text { Adelg } \\
\text { bifacial }\end{array}$ & $\begin{array}{c}\text { Red } \\
\text { bifacial }\end{array}$ & $\begin{array}{c}\text { Adel/red } \\
\text { unifacial }\end{array}$ & $\begin{array}{c}\text { Trab no } \\
\text { inv bif }\end{array}$ & $\begin{array}{c}\text { Bifacial } \\
\text { no dif }\end{array}$ & $\begin{array}{c}\text { Trab no } \\
\text { inv unif }\end{array}$ & $\begin{array}{c}\text { Unif } \\
\text { no dif }\end{array}$ & $\begin{array}{c}\text { Bipol } \\
(\mathbf{i} ?)\end{array}$ & Total \\
\hline QS3 & 26 & 25 & 2 & 9 & 4 & 24 & 6 & 7 & 103 \\
\hline PP4 & 17 & 13 & 1 & 7 & 23 & 115 & 33 & - & 209 \\
\hline PCh1.1 & 15 & 7 & 2 & 9 & 3 & 51 & 5 & 1 & 93 \\
\hline CChM1 & 3 & 20 & - & 156 & 9 & 109 & 61 & - & 358 \\
\hline
\end{tabular}

Cuadro 3. Cantidades de artefactos formatizados por clase técnica. 


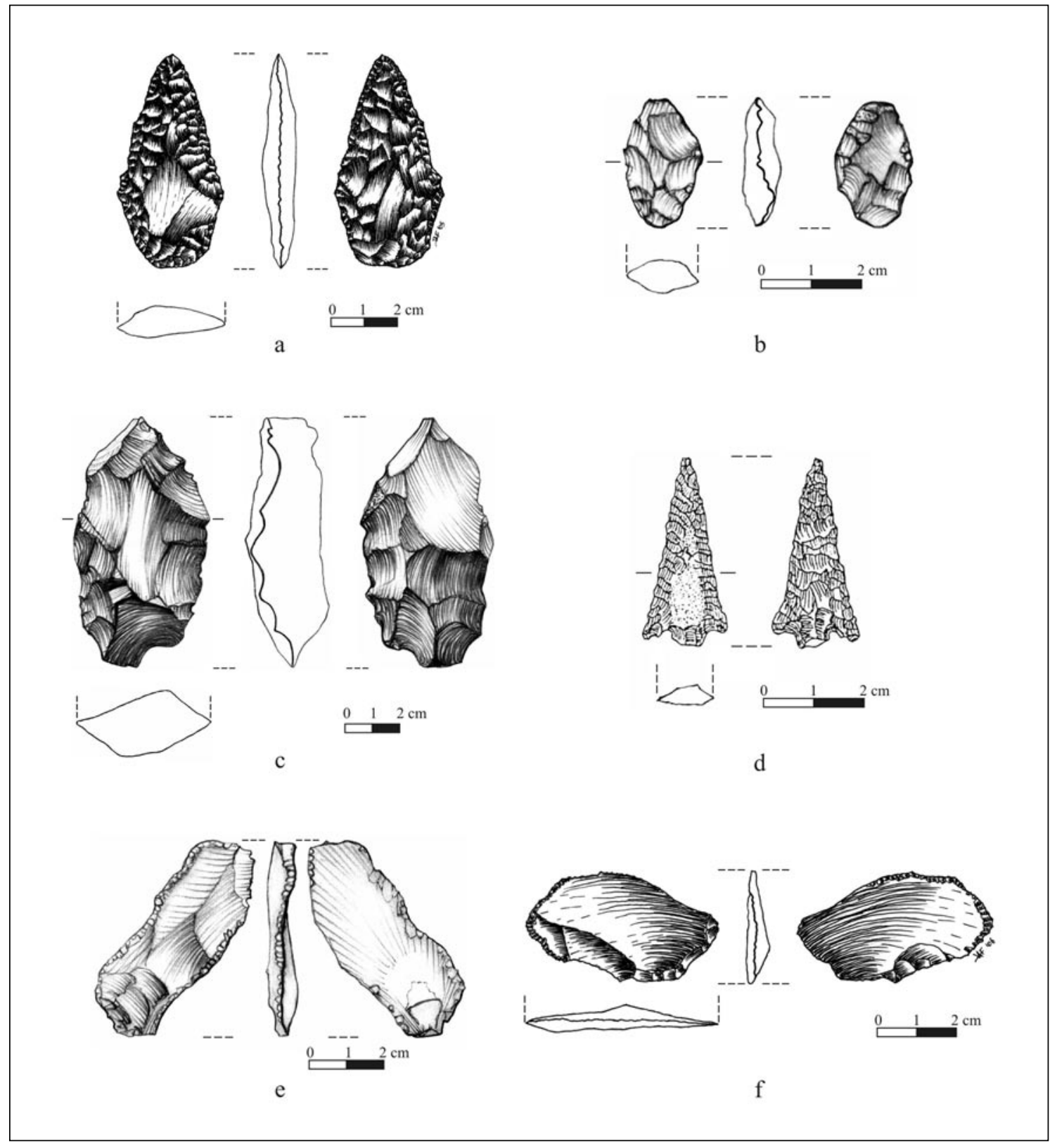

Figura 3. Ejemplos de clases técnicas en artefactos formatizados: a y b) Adelgazamiento bifacial; c y d) Reducción bifacial; e y f) Trabajo no invasivo unifacial y biifacial: a. QS3 - n 58; b. PCh1.1 - n 54; c. QS3 - n 316; d. CChM1 (tomado de Escola 1991); e. QS3 - nº 639 y; f. QS3 - n 31 .

necesario: a) generar una plataforma centrada con el plano de simetría o de equilibrio longitudinal (Inizan et al. 1999), ya que así se pueden lograr extracciones lo suficientemente largas, en una o en ambas caras, como para sobrepasar el centro de la/s misma/s, y 2) que los golpes contra la plataforma se den con ángulos extremadamente obtusos, de forma de producir lascas largas. Estos dos requerimientos del adelgazamiento le imponen una carga adicional de destreza técnica, necesitándose de un mayor control por parte del tallador (Whittaker 1994; Stout 2002). 


\section{El caso arqueológico: Lo que señalan las evidencias}

$\mathrm{Al}$ analizar las frecuencias de artefactos formatizados en los sitios arqueológicos considerados (Figura 4), es posible advertir la coexistencia de una serie de alternativas tecnológicas con distinto grado de inversión de trabajo en la formatización de artefactos, así como una fluctuación en las frecuencias de las mismas a lo largo del tiempo.

De esta forma, se registra que en los artefactos formatizados confeccionados por adelgazamiento bifacial hay una tendencia a un marcado decrecimiento que involucra la virtual desaparición de esta clase técnica hacia los 2000 AP, ya que se pasa de un $25 \%$ a un $1 \%$, aproximadamente, de representación en los conjuntos. Destaca, también, la disminución sustancial en la cantidad de bifaces en el GC2 respecto del GC1. Así, el primer grupo cronológico mencionado está formado por la sumatoria de los bifaces de dos sitios (PP4, n=17; PCh1.1, n=15), mientras que el último grupo, por los bifaces de un solo sitio (QS3, $\mathrm{n}=26)$. Estas tendencias son corroboradas al analizar los porcentajes de las lascas de adelgazamiento bifacial en muestras de desechos de talla (Hocsman 2004 Ms).

En el caso de aquellos instrumentos producidos por reducción bifacial, por su parte, se observa también una marcada disminución al pasar del GC1 al GC2, para posteriormente mantenerse con frecuencia relativamente constante.

En concordancia con el adelgazamiento bifacial, literalmente desaparecen el adelgazamiento y la reducción unifacial en los artefactos formatizados hacia los 3200 AP.

Ahora bien, de la correlación de estas clases técnicas que involucran el tratamiento de la o las caras, surge que el adelgazamiento bifacial prevalece

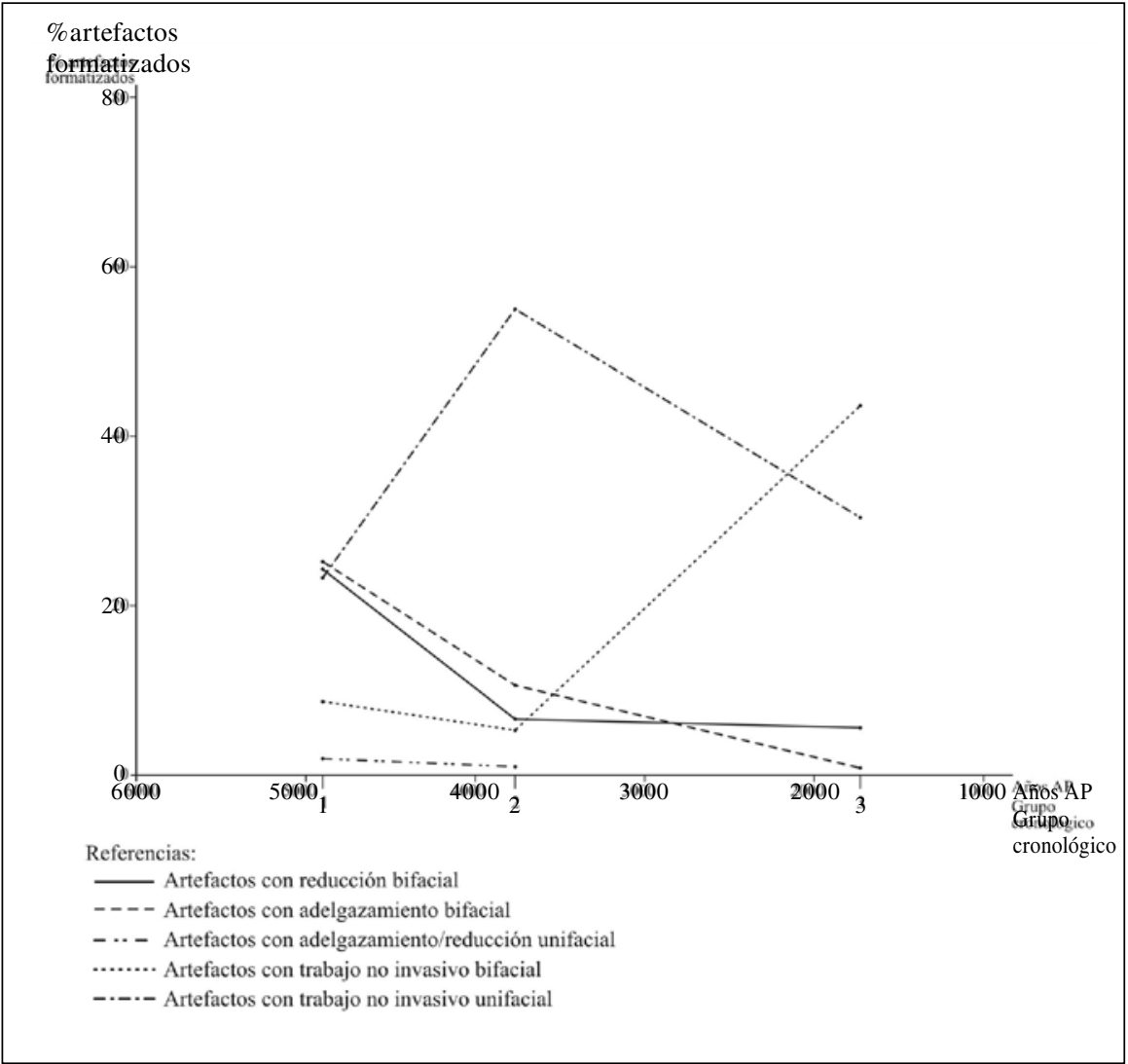

Figura 4. Tendencias temporales de clases técnicas en artefactos formatizados. 
levemente sobre la reducción bifacial hasta los 3200 AP, para prácticamente desaparecer hacia el 2000 AP, invirtiéndose dramáticamente la relación. Si a esto se suma la ausencia del adelgazamiento y de la reducción unifacial, es claro que la reducción bifacial no sólo es la única que continúa, sino que, además, ve incrementado notoriamente su papel, el cual está orientado a la confección de puntas de proyectil con limbo triangular de tipo isósceles, pedúnculo diferenciado y aletas entrantes (Escola 2000).

En lo que respecta al trabajo no invasivo bifacial parecería haber, a primera vista, una leve disminución en la frecuencia de esta clase técnica del GC1 al GC2 -de un 9\% a un 5\%, aproximadamente-, así como un importante incremento en el GC3 en relación al GC2, de un 5\% a un 44\%. Sin embargo, ya que el marcado aumento a partir del 2000 AP es consecuencia, en realidad, de una marcada sobrerrepresentación debido al elevado porcentaje de fragmentos de palas y/o azadas en CChM1 (Escola 2000), la tendencia podría ser tanto un franco decrecimiento en el tiempo como un aumento de escasa proporción durante el GC3. Es interesante denotar que los artefactos formatizados confeccionados por trabajo no invasivo bifacial tienen en este último momento un papel emergente, ya que se asocian exclusivamente a palas y/o azadas, artefactos sin correlato previo en el ámbito local.

Al considerar el trabajo no invasivo unifacial, finalmente, se evidencia un destacado incremento al pasar de $\mathrm{GC} 1$ al GC2, para posteriormente decrecer de GC2 al GC3 -del 23 al 55\% y del 55 al 30\%-, respectivamente. Más allá de tales fluctuaciones marcadas, es claro que esta clase técnica tuvo un rol preponderante a lo largo de toda la secuencia ocupacional, dadas sus frecuencias ampliamente mayoritarias en relación a las clases restantes, con excepción del GC1, donde se presenta prácticamente en igualdad de proporción con el adelgazamiento y la reducción bifacial. No obstante, este tipo de trabajo no invasivo ve acrecentada su importancia relativa con el paso del tiempo, ante la disminución de las frecuencias de artefactos formatizados confeccionados por adelgazamiento y reducción e, inclusive del trabajo no invasivo bifacial, es decir, se habría potenciado a expensas de estos últimos. En este sentido, es de destacar que para el 2000 AP prácticamente todo el repertorio de artefactos formatizados se confeccionaba de esta manera, quedando exceptuadas las puntas de proyectil y las palas y/o azadas.
Tales variaciones en las frecuencias artefactuales pueden ser entendidas como el resultado de procesos de toma de decisiones, donde las elecciones de los grupos humanos que ocuparon Antofagasta de la Sierra entre los 5500 y 1500 años AP implicaron, en la cuenta larga, una tendencia a invertir un menor esfuerzo en la manufactura de artefactos formatizados tallados con el paso del tiempo. En efecto, se produjo la desaparición del adelgazamiento y de la reducción unifacial, el adelgazamiento bifacial se hizo prácticamente inexistente y se mantuvo la reducción bifacial, pero sólo vinculada a la confección de puntas de proyectil; simultáneamente, se registra un virtual reemplazo de dichas clases técnicas por el trabajo no invasivo bifacial y unifacial, siendo preponderante este último. De esta forma, se optó por estrategias tecnológicas con bajos costos de producción en cuanto a requerimientos de tiempo, energía y destreza técnica (Pintar 1996; Escola 2000).

\section{Evidencias de cambio tecnológico en otras áreas de la porción meridional de los Andes Centro Sur}

Si bien no se cuenta, hasta el momento, con información específica acerca de las variaciones de las clases técnicas en sitios arqueológicos de la Puna Septentrional y su borde, así como del borde de puna en el desierto del norte de Chile, ubicados temporalmente entre los 5000 y 2000 años AP, es factible emplear información édita e inédita de modo de analizar, preliminarmente, si la tendencia a invertir un menor esfuerzo en la manufactura de artefactos formatizados, establecida para Antofagasta de la Sierra, se limitaba al ámbito local, o bien, tenía carácter regional.

En general, los trabajos considerados señalan que los conjuntos de artefactos formatizados de distintas cronologías están formados por piezas con distinto grado de inversión de trabajo, diferenciando, por un lado, artefactos formatizados de baja inversión de tiempo y energía, manufacturados por trabajo unifacial marginal y, por el otro, piezas con mayor inversión de trabajo, bifaciales, generalmente puntas de proyectil y preformas de las mismas (p.e., Núñez 1992, 1994; Lavallée et al. 1997; García 1998; Yacobaccio et al. 1999).

Más allá de esto, las muestras están compuestas mayoritariamente por instrumentos confeccionados con bajos costos de producción, como lo atestiguan 
las ocupaciones del sitio Tomayoc, datadas entre 3500 y 3200 años AP (Lavallée et al. 1997), localizado en la Sierra del Aguilar, o del Alero Unquillar, fechadas hacia los 3500 años AP (Yacobaccio et al. 2000), situado en Susques, en la Puna Septentrional argentina. Se destaca aquí la coincidencia con la secuencia de Antofagasta de la Sierra. Al igual que en esta última, además, la información disponible permitiría sostener una tendencia a invertir menor tiempo y energía en la producción de artefactos tallados con el paso del tiempo a nivel regional. En este sentido pueden señalarse las conclusiones alcanzadas por Carrasco (2004) en cuanto a una disminución en la inversión de trabajo al analizar una serie de sitios adscribibles al Arcaico Tardío, Formativo Temprano, Formativo Tardío, Intermedio Tardío y Tardío de la localidad de Caspana o las afirmaciones coincidentes de Rees y de Souza (2004) al abordar el Período Formativo en la Subregión del río Salado, a una escala espacial más grande en relación con el primero, en el ambiente desértico del norte chileno.

Esta tendencia surge, también, de la comparación de los sitios Tulan-52 y Puripica-1 (Núñez 1981), por un lado, y Tulan-54 (Núñez 1994), por el otro, localizados en las quebradas de Tulan y Puripica, en el desierto del norte de Chile. Antes de pasar al caso concreto, es pertinente destacar que la adscripción a clases técnicas planteada es preliminar, ya que está basada sólo en las descripciones tipológicas de las categorías de artefactos formatizados realizadas por Núñez y su equipo y no sobre los artefactos en sí. No obstante, se considera viable su uso si se lo toma como una aproximación gruesa, como es este caso.

Así, los dos primeros sitios, datados entre los 5000 y 4000 años AP muestran estrechas coincidencias en la representación de las piezas formatizadas por adelgazamiento/reducción bifacial y unifacial en relación a aquellas confeccionadas por trabajo no invasivo bifacial y unifacial, siendo de $34 \%$ y $66 \%$, respectivamente, en Tulan-52 (datos tomados de Núñez 1981), y de $33.80 \%$ y $66.20 \%$, respectivamente, en Puripica-1 (a base de datos de Núñez et al. 1999). Estas diferencias de porcentaje entre dichas categorías generales se profundizan al incluir al sitio Tulan-54, fechado entre los 3200 y 2600 años AP, ya que pasan a un $7.82 \%$ y a un $90.42 \%$, respectivamente (datos tomados de Núñez 1994), señalando la misma tendencia general observada en Antofagasta de la Sierra de preponderancia relativa del trabajo no invasivo con el paso del tiempo.

Ahora bien, de acuerdo a observaciones preliminares de materiales líticos de los sitios Tulan-52 y Tulan-54, surgen dos aspectos de interés claramente disímiles a lo ocurrido en Antofagasta de la Sierra, relacionados con las frecuencias de aparición del adelgazamiento y de la reducción bifacial. En primer lugar, la importancia relativa de la reducción bifacial sobre el adelgazamiento bifacial en Tulan-52, situación inversa a la de los GC1 y GC2 y, en segundo lugar, la presencia de adelgazamiento bifacial en Tulan-54 en proporciones significativas, cuando en el GC3 prácticamente había desaparecido.

Este último punto es importante, ya que implica que el adelgazamiento bifacial estaba plenamente vigente en un contexto agropastoril inicial datado hacia los 3000 AP, lo cual se ve reforzado por la presencia de esta clase técnica en contextos agropastoriles tempranos de la Puna Septentrional argentina. Ejemplo de esto son los artefactos recuperados en Cueva de Cristóbal, con ocupaciones datadas entre $2860 \pm 160$ AP y $2530 \pm 100$ AP (Fernández 1988/89), cuyos materiales incluyen artefactos formatizados confeccionados de esta forma (al menos 10 piezas de acuerdo a descripciones y fotografías) y lascas de adelgazamiento bifacial (53.96\% del total de desechos). También pueden mencionarse las lascas de adelgazamiento bifacial, con una representación del $11.2 \%$ (sobre cuarcita), de las capas 5 y 6 del sitio Inca Cueva alero 1, fechada la primera en $2900 \pm 70$ AP (García 1998).

Esto significaría que la desaparición de esta clase técnica se produjo en el seno de las sociedades productoras puneñas y no durante la transición de cazadores recolectores a grupos agropecuarios, entre los 3000 y 2500 años AP, aproximadamente.

Al respecto, se toma como límite más tardío esta fecha, ya que las evidencias de adelgazamiento bifacial tienden a desaparecer para estos momentos, de acuerdo a información recuperada en sitios datados entre 2500 y 2000 años AP de la puna argentina y su borde, tales como: Las Cuevas, Cerro el Dique y Potrero Grande (Raffino 1977), en la quebrada

\footnotetext{
5 Lo cual fue posible sólo gracias al total acceso brindado por L. Núñez a las colecciones de ambos sitios y a la colaboración de P. de Souza.
} 
del Toro, o Campo Colorado (Tarragó 1996), en el Alto Valle Calchaquí, todos situados en el borde oriental de la puna salteña; Cochinoca-39, en Susques, en la puna jujeña (Fernández Distel 1998); y Estancia Grande, en la quebrada de Humahuaca, en el borde oriental de la Puna Jujeña (Palma y Olivera 1992/93).

Entonces, para el 2500 AP, continuaría sólo la reducción bifacial, empleada específicamente en la confección de las puntas de proyectil de limbo triangular con pedúnculo diferenciado y aletas entrantes (Escola 1987, 1991). Si a esto se suma la desaparición de la reducción unifacial, ampliamente extendida en sitios de la puna argentina con anterioridad a los 3000 AP y cuyo artefacto arquetípico serían las llamadas "puntas Saladillo" (Fernández 1996), resulta en una mayor importancia relativa del trabajo no invasivo en los conjuntos con el paso del tiempo.

Sin lugar a dudas, para validar las tendencias aquí señaladas se requiere no sólo de una recopilación de mayores casos, sino también de un análisis pormenorizado de las muestras sobre la base de una caracterización por clases técnicas. En este marco, se podría sugerir, como hipótesis, que la disminución en la inversión de trabajo en los artefactos tallados fue un proceso relativamente ubicuo en el altiplano de la porción meridional de los Andes Centro Sur, más allá de las particularidades regionales.

\section{Consideraciones finales}

Tal como fue señalado previamente, las soluciones tecnológicas son las consecuencias particulares de decisiones que buscan resultados óptimos de objetivos identificados por los actores. Es así que, bajo ciertas condiciones relacionadas básicamente a necesidades físicas, como la subsistencia, se van a priorizar aquellas estrategias u opciones que cumplan mejor los objetivos propuestos que otras existentes. En este marco, se discuten tendencias diacrónicas en estrategias de producción de artefactos formatizados tallados a partir de la observación de trayectorias de cambio en la conformación de conjuntos de instrumentos espacial y temporalmente acotados.

De esta forma, los grupos humanos que ocuparon Antofagasta de la Sierra entre los 5500 y 1500 años AP contaban con una serie de opciones tecnológicas con distintos costos de producción y requerimientos de destreza técnica, cuya representación fue variando con el transcurso del tiempo. Así, destacan, por un lado, la disminución en la frecuencia de artefactos con adelgazamiento y con reducción bifacial, y, por el otro, el aumento de artefactos con trabajo no invasivo, básicamente unifacial, con el correr del tiempo. Estas tendencias implicaron una inversión de trabajo decreciente en la manufactura, pasando de un mayor a un menor grado de modificación de la morfología de los instrumentos, lo que significaría que las poblaciones en transición de Antofagasta de la Sierra y posiblemente de otras áreas de los Andes eligieron, a partir de la evaluación de sus costos y beneficios, alternativas tecnológicas específicas.

Así, estos grupos humanos puneños habrían desarrollado y modificado sus estrategias tecnológicas en función de una toma de decisiones que tendría por objetivo maximizar la tasa de retorno de actividades específicas (Bettinger 1991), sopesando sus opciones y optando por aquellas que proveían la razón costo-beneficio más alta, dados costos de oportunidad determinados (Kelly 2000). Como cada una de las alternativas tecnológicas en juego se asociaría a una tasa de retorno particular (Bousman 1993; Kelly 1995), es evidente que dichas tasas de retorno sufrieron variaciones que llevaron a que ciertas clases técnicas cayeran en desuso y que otras pasaran a ser redituables.

Un punto importante a tener en cuenta refiere a la naturaleza de lo "óptimo". Así, al hablar de optimización, no significa que todos los comportamientos son óptimos, es decir, que las elecciones necesariamente resultarán en la mejor opción posible, sino que habrá una "tendencia hacia" la optimización (Jochim 1983; Bettinger 1991; Kelly 1995).

Ahora bien, el comportamiento tecnológico implica la solución de problemas ante situaciones generadas por el ambiente físico y social (Nelson 1991). El hecho de que ciertas variantes tecnológicas se vieran favorecidas sobre otras lleva a considerar qué fue lo que desencadenó el cambio. Al respecto, hay una serie de factores, para el caso de Antofagasta de la Sierra, que podrían haber tenido un papel importante en este sentido, afectando la toma de decisiones y, por ende, la representación de las clases técnicas. Entre estos se encuentran los efectos de la movilidad reducida sobre la estructuración de los conjuntos líticos y las variaciones del riesgo de subsistencia producto de cambios climáticos, o bien, 
de la puesta en práctica de actividades productivas, ya sean agrícolas y/o pastoriles. También deberían considerarse los efectos de los problemas vinculados con la programación de actividades que compiten entre sí o que son mutuamente exclusivas, como son, en efecto, la producción agrícola y la pastoril (ver Escola 1996, 2000).

Al tratar con la secuencia paleoclimática local, generada recientemente por Olivera y colaboradores (2002 Ms), se observa que desde los 6500/6000 AP las condiciones ambientales eran marcadamente áridas, situación que cambia hacia el 3000 AP cuando se instala un clima húmedo, que se extiende hasta el 1600 AP aproximadamente. Lo expresado permitiría postular que, entre el 6000 y el 3000 AP, los grupos humanos debían atenerse a una baja predictibilidad (y accesibilidad) de los recursos bióticos, con el consecuente aumento del riesgo. El mejoramiento de las condiciones locales a partir del 3000 AP habría tenido como consecuencia, por un lado, un marco de mayor estabilidad y, por el otro, el aumento de la extensión de las áreas de pastura y, por ende, de las poblaciones de camélidos silvestres. Se destaca, al respecto, la correlación sugerida por Torrence (1989) de los artefactos con inversión de trabajo extra en su manufactura, como los instrumentos con adelgazamiento bifacial, con la severidad del riesgo.

Pero la disminución del riesgo en Antofagasta de la Sierra no sólo puede ser relacionada con variaciones climáticas, sino también con la puesta en práctica de actividades productivas, agrícolas y pastoriles (Pintar 1996; Escola 2000; Babot 2004). Si se parte de un continuum donde en un extremo están los cazadores recolectores, que se enfrentan a una distribución espacial y temporal de los recursos explotados (presas) dada por la naturaleza y, en el otro, a agricultores y pastores que controlan los recursos a través del manejo de su reproducción, hay diferencias notorias entre unos y otros en cuanto al riesgo de subsistencia involucrado (Torrence 1989), siendo mucho menor en el segundo caso, aunque indudablemente presente (Escola 1996).

Diversas clases de vestigios arqueológicos brindan evidencias de una reducción de la movilidad residencial en Antofagasta de la Sierra en el lapso considerado (Hocsman 2002). Así, Aschero y colaboradores (1993/94) señalan, para el lapso 5500-3500 AP, la existencia en la microrregión de una movilidad muy pautada, con retorno a lugares previstos, en el marco de un sistema de asentamiento semisedentario, o bien, de recorridos/circuitos estacionales bien demarcados. Un alto grado de sedentarismo, con asentamientos con ocupación de año redondo, se presentaría recién hacia el 2000 AP, con la aparición de bases residenciales de actividades múltiples asociadas a grupos agropastoriles plenos (Olivera 1992).

Al conjugar estos factores causales surge que, amén de estar posiblemente interrelacionados, se evidencian problemas de equifinalidad, ya que cada uno de los mismos generaría la misma tendencia en el registro arqueológico (Hocsman 2002). Más allá de esto, se considera, en definitiva, que los cambios en la frecuencia de los instrumentos se relacionan con la disminución de la movilidad residencial, las variaciones paleoclimáticas y/o el cambio en la subsistencia.

Así, los artefactos que mejor cumplían las nuevas condiciones aumentaron su representación a través del tiempo. Se destaca que otros factores de diversa índole también pueden haber actuado. Al respecto, ver, por ejemplo, las consideraciones de Rees y de Souza (2004) para la Subregión del río Salado en la cuenca del Loa (Chile).

Finalmente, el análisis llevado a cabo permitió identificar, por un lado, una serie de coincidencias entre las áreas abordadas como, por ejemplo, la desaparición del adelgazamiento y la reducción unifacial hacia el $3000 \mathrm{AP}$ y, por el otro, interesantes diferencias que requieren ser exploradas con mayor profundidad en el futuro, por ejemplo, en relación con las frecuencias inversas del adelgazamiento y la reducción bifacial al comparar Antofagasta de la Sierra con los sitios chilenos considerados, en el lapso 5500-3200 AP.

Si bien tales diferencias serían esperables atendiendo a particularidades locales, el hecho de tratarse de un momento transicional potencia la posibilidad de una persistencia diferencial de las alternativas tecnológicas. Deben considerarse, además, las variaciones producto de las características de las secuencias arqueológicas locales. Un caso de esto podría ser el vacío de información en la secuencia de Antofagasta de la Sierra en cuanto a sitios agropastoriles iniciales, en relación con otras áreas de la Puna de Atacama, que tiene por correlato las frecuencias mínimas del adelgazamiento bifacial en contextos agropastoriles plenos del 2000 AP. 
Diversos autores han señalado la continuidad entre los grupos cazadores recolectores y las sociedades agropastoriles que ocuparon la porción meridional de los Andes Centro Sur, desde distintas líneas de evidencia (Aschero 1994; Núñez 1994; Yacobaccio 1994). En el marco de lo aquí expresado, la tecnología lítica debería ser considerada también un elemento más de continuidad, como lo revela la perduración de clases técnicas específicas; sería el caso, por ejemplo, de la reducción bifacial.
Agradecimientos A Lidia Benítez de Parra, de la Cátedra de Estadística de la Facultad de Ciencias Naturales e I.M.L. (U.N.T.), por el asesoramiento brindado en el tratamiento estadístico. A Luis Guillermo Babot por el tratamiento digital de las imágenes. Este trabajo se realizó en el marco de los proyectos PIP-CONICET 3041, FONCYT-PICT 9888 y un programa CIUNT (2001-2003), dirigidos por Carlos Aschero.

\section{REFERENCIAS CITADAS}

ANDREFSKY, W., 1998. Lithics. Macroscopic approaches to analysis. Cambridge University Press, Cambridge.

ASCHERO, C., 1983 Ms. Ensayo para una clasificación morfológica de artefactos líticos aplicada a estudios tipológicos comparativos. Apéndices A-C. Revisión. Cátedra de Ergología y Tecnología, Facultad de Filosofía y Letras, Universidad de Buenos Aires, Buenos Aires.

1994. Reflexiones desde el Arcaico Tardío (6000-3000 AP). Rumitacana. Revista de Antropología 1: 13-17.

ASCHERO, C. y S. HOCSMAN, 2004. Revisando cuestiones tipológicas en torno a la clasificación de artefactos bifaciales. En Temas de arqueología, análisis lítico, A. Acosta, D. Loponte y M. Ramos (Eds.), pp. 7-25. Universidad Nacional de Luján, Luján.

ASCHERO, C., C. ELKIN y E.L.PINTAR, 1991. Aprovechamiento de recursos faunísticos y producción lítica en el Precerámico Tardío. Un caso de estudio: Quebrada Seca 3 (Puna Meridional argentina). Actas XII Congreso Nacional de Arqueología Chilena 2: 101-114. Santiago.

ASCHERO, C., L. MANZI y A. G. GOMEZ, 1993-94. Producción lítica y uso del espacio en el nivel 2b4 de Quebrada Seca-3. Relaciones de la Sociedad Argentina de Antropología XIX: 191-214.

BABOT, M. P., 2004. Tecnología y utilización de artefactos de molienda en el noroeste prehispánico. Tesis Doctoral. Facultad de Ciencias Naturales e Instituto Miguel Lillo. Universidad Nacional de Tucumán, Tucumán.

BAMFORTH, D. B., 1992. Quarries in context: A regional perspective on lithic procurement. En Stone tool procurement, production and distribution in California prehistory, vol. 2, pp.131-156. Institute of Archaeology Series, University of California, Los Angeles.

BECK, C., 1998. Projectile point types as valid chronological units. En Units issues in archaeology. Measuring time, space, and material, A. Ramenosfsky y A. Steffen (Eds.), pp. 21-40. The University of Utah Press, Salt Lake City.

BETTINGER, R. L., 1991. Hunter-gatherers: Archaeological and Evolutionary Theory. Plenum Press, Nueva York.
BLEED, P., 1986. The optimal design of hunting weapons: Maintainability or reliability. American Antiquity 51 (4): 737-747.

1997. Content as variability, result as selection: Toward a behavioral definition of technology. En Rediscovering Darwin: Evolutionary Theory and archeological explanation, C. M. Barton y G. A. Clark (Eds.), pp. 95-104. Archeological Papers of the American Anthropological Association 7, Plenum Press, Nueva York.

BOONE, J. L. y E. A. SMITH, 1998. It is evolution yet? A critique of evolutionary archaeology. Current Anthropologist 39: 141-173.

BOUSMAN, C. B., 1993. Hunter-gatherer adaptations, economic risk and tool design. Lithic Technology 18 (1 y 2): 59-86.

BORRERO, L. A., 1993. Artefactos y evolución. Palimpsesto 3: $15-32$.

BRADLEY, B. A. e Y. GIRIA, 1996. Concepts of the technological analysis of flaked stone: A case study from the High Arctic. Lithic Technology 21(1): 23-47

CARRASCO, C., 2004. Uso de tecnologías líticas entre el Arcaico Tardío y el Período Tardío: El modelo de la localidad de Caspana. Chungara vol. especial: 29-35.

COWAN, F. L., 1999. Making sense of flake scatters: Lithic technological strategies and mobility. American Antiquity 64 (4): 593-607.

DUNNELL, R. C., 1989. Aspects of the application of evolutionary theory in archaeology. En Archaeological thought in America, C. Lamberg-Karlovsky (Ed.), pp. 35-49. Cambridge University Press, Cambridge.

ESCOLA, P., 1987. Las puntas de proyectil del Formativo en puna y quebradas de acceso: Un estudio tecno-tipológico de cuatro casos de análisis. Tesis de Licenciatura. Facultad de Filosofía y Letras, Universidad de Buenos Aires, Buenos Aires.

1991. Puntas de proyectil en contextos formativos: Acercamiento tecno-tipológico a través de cuatro casos de análisis. Actas XI Congreso Nacional de Arqueología Chilena 2: 175-187. Santiago. 
1996. Riesgo e incertidumbre en economías agropastoriles: Consideraciones teórico metodológicas. Arqueología 6: 9-24.

2000. Tecnología lítica y sociedades agropastoriles tempranas. Tesis Doctoral, Facultad de Filosofía y Letras, Universidad de Buenos Aires, Buenos Aires.

FERNANDEZ, J., 1988/89. Ocupaciones alfareras (2860 \pm 160 años AP) en la cueva de Cristóbal, Puna de Jujuy, Argentina. Relaciones de la Sociedad Argentina de Antropología XVII (2): 139-178.

1996. Potrero de Caballo Muerto: Aspectos arqueológicos, cronológicos y paleoambientales del Precerámico Tardío en el ecosistema hidrófilo de las vegas puneñas. Actas y Memorias del XI Congreso Nacional de Arqueología Argentina (13 ${ }^{\mathrm{a}}$ Parte). Revista del Museo de Historia Natural de San Rafael XXV: (1/4): 23-51.

FERNANDEZ DISTEL, A., 1998. Arqueología del Formativo en la Puna Jujeña (1800 AC al 650 DC). Colección Mankacén, Buenos Aires.

GARCIA, L. C., 1998. Arqueología de asentamientos formativos en la Puna Oriental y su borde, Provincia de Jujuy. El cambio hacia una vida crecientemente sedentaria y productiva en Azul Pampa, Departamento de Humahuaca. Tesis Doctoral, Facultad de Filosofía y Letras, Universidad de Buenos Aires, Buenos Aires.

HAYDEN, B., N. FRANCO y J. SPAFFORD, 1996. Evaluating lithic strategies and design criteria. En Stone tools. Theoretical insights into human prehistory, G. Odell (Ed.), pp. 9-45. Plenum Press, Nueva York.

HOCSMAN, S., 2002. ¿Cazadores recolectores complejos en la Puna Meridional argentina? Entrelazando evidencias del registro arqueológico de la microrregión de Antofagasta de la Sierra (Catamarca). Relaciones de la Sociedad Argentina de Antropología XXVII: 193-214.

-2002 Ms. Producción lítica, variabilidad y cambio en Antofagasta de la Sierra (ca. 5500- 2000 AP): La transición hacia las sociedades agropastoriles. Informe de Avance CONICET (2001-2003)

-2004 Ms. Tecnologías líticas extractivas en bases residenciales de cazadores recolectores y grupos agropastoriles: Una comparación. Actas del XIV Congreso Nacional de Arqueología Argentina. Rosario. En prensa.

INIZAN, M. L., M. REDURON-BALLINGER, H. ROCHE y J. TIXIER, 1999. Technology and terminology of knapped stone. Préhistoire de la Pierre Taillée, Tome 5. Cercle de Recherches et d'Etudes Préhistoriques, Nanterre.

JOCHIM, M. A., 1976. Hunter-gatherer subsistence and settlement. A predictive model. Academic Press, Londres.

1983. Optimization models in context. En Archaeological hammers and theories, J. Moore y A. Keene (Eds.), pp. 157172. Academic Press, Nueva York.
KELLY, R. L., 1995. The foraging spectrum: Diversity in hunter-gatherer lifeways. Smithsonian Institution Press, Washington D. C.

2000. Elements of a behavioral ecological paradigm for the study of prehistoric hunter-gatherers. En Social theory in archaeology, M. Schiffer (Ed.), pp. 63-78. The University of Utah Press, Salt Lake City.

LAVALLEE, D., M. JULIEN, C. KARLIN, L. GARCIA, D. POZZI-ESCOT y M. FONTUGNE, 1997. Entre desierto y quebrada: Tomayoc, un alero de la puna. Avances en Arqueología 3: 9-39.

LOPEZ CAMPENY, S. M., 2001 Ms. Actividades domésticas y organización del espacio intrasitio. El sitio Punta de la Peña 9 (Antofagasta de la Sierra, Prov. de Catamarca). Trabajo final de la carrera de Arqueología, Facultad de Ciencias Naturales e I.M.L, Universidad Nacional de Tucumán, San Miguel de Tucumán.

LYMAN, R. L. y M. J. O'BRIEN, 1998. The goals of evolutionary archaeology. History and explanation. Current Anthropology 39 (5): 615-652.

NELSON, M., 1991. The study of technological organization. Journal of Archaeological Method and Theory 3: 57-100.

NELSON, M. y H. LIPPMEIER, 1993. Grinding stone design as conditioned by land-use pattern. American Antiquity 58: 286-305.

NUÑEZ, L., 1981. Asentamientos de cazadores recolectores tardíos de la Puna de Atacama: Hacia el sedentarismo. Chungara 8: 137-168.

1992. Ocupación arcaica en la Puna de Atacama: Secuencia, movilidad y cambio. En Prehistoria sudamericana. Nuevas perspectivas, B. Meggers (Ed.), pp. 283-307. Taraxacum, Washington D. C.

_ 1994. Emergencia de complejidad y arquitectura jerarquizada en la Puna de Atacama: Las evidencias del sitio Tulan-54. En Taller de Costa a Selva, M. Albeck (Ed.), pp. 85-108. Instituto Interdisciplinario Tilcara, Buenos Aires.

NUÑEZ, L., M. GROSJEAN e I. CARTAJENA, 1999. Un ecorrefugio oportunístico en la Puna de Atacama durante eventos áridos del Holoceno Medio. Estudios Atacameños 17: $125-174$.

OLIVERA, D., 1992. Tecnología y estrategias de adaptación en el Formativo (Agroalfarero Temprano) de la Puna Meridional argentina. Un caso de estudio: Antofagasta de la Sierra (Provincia de Catamarca). Tesis Doctoral, Facultad de Ciencias Naturales, Universidad Nacional de La Plata, La Plata.

OLIVERA, D., P. TCHILINGUIRIAN y M. J. DE AGUIRRE, 2002 Ms. Cultural and environmental evolution in the meridional sector of the Puna of Atacama during the Holocene. En XIV International Congress of Prehistoric and Protohistoric Sciences. B.A.R. International Press, Bélgica. En prensa. 
PALMA, J. R. y D. OLIVERA, 1992/93. Hacia la contrastación de un modelo arqueológico para el Formativo Regional en Humahuaca: El caso de Estancia Grande. Cuadernos del Instituto Nacional de Antropología y Pensamiento Latinoamericano 14: 237-259.

PINTAR, E. L., 1996. Prehistoric holocene adaptations to the Salt Puna of Northwestern Argentina. Tesis Doctoral, Graduate Faculty of Dedman College, Southern Methodist University, Tulsa.

RAFFINO, R. A., 1977. Las aldeas del Formativo Inferior de la quebrada del Toro (Salta, Argentina). Estudios Atacameños 6: 64-108.

REES, C. y P. DE SOUZA, 2004. Producción lítica durante el Período Formativo en la Subregión del río Salado. Chungara vol. especial: 453-465.

SCHIFFER, M., 1995. Behavioral archaeology: First principles. University of Utah Press, Salt Lake City.

SCHIFFER, M. y J. M. SKIBO, 1987. Theory and experiment in the study of technological change. Current Anthropology 28: 595-622.

1997. The explanation of artifact variability. American Antiquity 62 (1): 27-50.

SMITH, E. y B. WINTERHALDER, 1992. Natural selection and decision making: Some fundamental principles. En Evolutionary ecology and human behavior, E. Smith y B. Winterhalder (Eds.), pp. 25-60. Aldine de Gruyter, Nueva York.

STOUT, D., 2002. Skill and cognition in stone tool production. An ethnographic case study from Irian Jaya. Current Anthropology 43 (5): 693-722.

TARRAGO, M., 1996. El Formativo en el Noroeste Argentino y el Alto Valle Calchaquí. Arqueología del Temprano en el Noroeste Argentino. Revista del Museo de Historia Natural de San Rafael XXIII (1/4): 103-119.

TOMKA, S. A., 2001. The effect of processing requirements on reduction strategies and tool form: A new perspective. En Lithic debitage. Context, form, meaning, W. Andrefsky (Ed.), pp. 207-223. The University of Utah Press, Salt Lake City.
TORRENCE, R., 1989. Retooling: Towards a behavioral theory of stone tools. En Time energy and stone tools, R. Torrence (Ed.), pp. 57-66. Cambridge University Press, Cambridge.

TOSELLI, A., 1998 Ms. Selección de materias primas líticas y organización tecnológica en el sitio Punta de la Peña 4 (PP4), Depto. Antofagasta de la Sierra, Prov. de Catamarca. Trabajo Final de la carrera de Arqueología. Facultad de Ciencias Naturales e Instituto Miguel Lillo. Universidad Nacional de Tucumán, San Miguel de Tucumán.

WINTERHALDER, B., 2001. The behavioural ecology of hunter-gatherers. En Hunter-gatherers: An interdisciplinary perspective, C. Panter-Brick, R. Layton y P. Rowley-Conwy (Eds.), pp. 12-38. Cambridge University Press, Cambridge.

WINTERHALDER, B. y E. SMITH, 1992. Evolutionary ecology and the social sciences. En Evolutionary ecology and human behavior, E. Smith y B. Winterhalder (Eds.), pp. 3-23. Aldine de Gruyter, Nueva York.

WHITTAKER, J. C., 1994. Flintknapping. Making and understanding stone tools. University of Texas Press, Austin.

YACOBACCIO, H., 1994. Hilos conductores y nudos gordianos: Problemas y perspectivas en la arqueología de cazadores recolectores puneños. Rumitacana. Revista de Antropología 1: 19-21.

1998. The evolution of South Andean hunter-gatherers. International Union of Prehistoric and Protohistoric Sciences. Proceedings of the XIII Congress, vol. 5: 389-394. A.B.A.C.O. Edizioni, Forli.

YACOBACCIO, H. y A. G. GURAIEB, 1994. Tendencia temporal de contextos arqueológicos: Area del río Pinturas y zonas vecinas. En Contribución a la arqueología del río Pinturas. Provincia de Santa Cruz, C. Gradín y A. Aguerre (Eds.), pp. 13-28. Búsqueda de Ayllu, Concepción del Uruguay.

YACOBACCIO, H., M. LAZZARI, A. G. GURAIEB y G. IBAÑEZ, 2000. Los cazadores en el borde oriental de Atacama (Susques, Jujuy). Revista Arqueología 10: $11-38$. 